\title{
Intellectual Disability, Falls and Gait Disturbances: A Misdiagnosis
}

\author{
Noel Lorenzo Villalba ${ }^{1}$, Santiago Díaz Nicolas², Maria Belen Alonso Ortiz ${ }^{3}$, Zaida Cordoba Sosa ${ }^{4}$, \\ Saturnino Suárez Ortega ${ }^{3}$, Abrar-Ahmad Zulfiqar ${ }^{1}$ \\ ${ }^{1}$ Service de Médecine Interne, Diabète et Maladies Métaboliques, Hôpitaux Universitaires de Strasbourg, Strasbourg, France \\ 2 Neurology Department, Dr Negrin University Hospital, Las Palmas de Gran Canaria, Spain \\ ${ }^{3}$ Internal Medicine Department, Dr Negrin University Hospital, Las Palmas de Gran Canaria, Spain \\ ${ }^{4}$ Internal Medicine Department, Fuerteventura General Hospital, Puerto del Rosario, Spain
}

Received: $12 / 01 / 2020$

Accepted: $16 / 01 / 2020$

Published: $13 / 02 / 2020$

How to cite this article: Lorenzo Villalba N, Díaz Nicolas S, Alonso Ortiz MB, Cordoba Sosa Z, Suárez Ortega S, Zulfiqar AA. Intellectual disability, falls and gait disturbances: a misdiagnosis. EJCRIM 2020;7: doi:10.12890/2020_001488.

Conflicts of Interests: The Authors declare that there are no competing interests.

This article is licensed under a Commons Attribution Non-Commercial 4.0 License

\section{ABSTRACT}

We report the case of a 27 -year-old man presenting with slowly progressive extrapyramidal dysfunction and learning disability considered to have a syndromic intellectual disability. The re-evaluation of the clinical features and the investigations performed led to the diagnosis of atypical pantothenate kinase-associated neurodegeneration (PKAN).

\section{LEARNING POINTS}

- Patients with an intellectual disability should be carefully evaluated.

- In the evaluation of a patient with extrapyramidal dysfunction for several years, with gradual progression, spasticity and psychiatric disturbances, PKAN should be considered.

\section{KEYWORDS}

Neurodegeneration with brain iron accumulation, pantothenate kinase-associated neurodegeneration, magnetic resonance imaging

\section{INTRODUCTION}

Neurodegeneration with brain iron accumulation (NBIA) describes a heterogeneous family of inherited diseases characterized by brain iron accumulation, particularly in the basal ganglia. Prevalence data are incomplete, but all forms of NBIA are considered to be "ultra-rare" with fewer than $1 / 1,000,000$ individuals affected ${ }^{[1]}$. The most common manifestation of childhood NBIA is classic pantothenate kinaseassociated neurodegeneration (PKAN), a severe, progressive type of autosomal recessive neuroaxonal dystrophy characterized by the early onset of symptoms (as opposed to atypical PKAN, with an average age of onset of 14 years). Onset of the disease in adulthood is rarely seen, the clinical presentation of which is completely different.

\section{CASE DESCRIPTION}

The patient was a 27-year-old man presenting with gait disturbance and falls. Some episodes of unmotivated laughing, and problems keeping up in school and needing support, were also referred to. There was no tremor, bradykinesia or other symptoms. Past medical history was relevant for an episode of moderate hypoglycaemia 2 hours after birth and normal psychomotor development until he was 5 years of age when his mother remarked that the patient sometimes had difficulties in terms of remaining straight in a chair, handling objects and falls. Difficulty with self-help and self-care skills was also noted. In addition, gait problems started at age 8 when he was seen by an orthopaedic 
specialist without any diagnosis. He was evaluated by a psychiatrist who considered the patient to present a syndromic intellectual disability. The family history was irrelevant.

The physical examination revealed and intellectual coefficiency border-line. Cranial nerves, muscle strength, muscle tone and osteotendinous reflexes were normal. The finger-to-nose test was normal. No bradykinesia or tremor were noted. However, a slight troncular stiffness and dystonic posture of the left foot were found. The cardiorespiratory and abdominal examinations were unremarkable.

Laboratory investigations showed a normal complete blood count, normal electrolytes and normal renal function. Liver, coagulation and thyroid function test results were within the normal range. The chemistry panel was completed by the determination of ferritin, copper, ceruloplasmin, serum phenylalanine and long-chain fatty acids, without any abnormal results. In this setting, a karyotype study was performed which excluded fragile X syndrome. Magnetic resonance imaging (MRI) showed bilateral, symmetric foci of T2 hyperintensity in the globus pallidus (GP) surrounded by pallidal T2 hypointensity (Fig. 1), the so-called "Eye-of-the-tiger" sign. A genetic panel for NBIA, specifically PKAN, was ordered for this patient, including pantothenate kinase 2 (PANK2) testing - the genetic marker for pantothenate kinase 2 (PanK2) enzyme. This showed a PANK2 gene mutation.

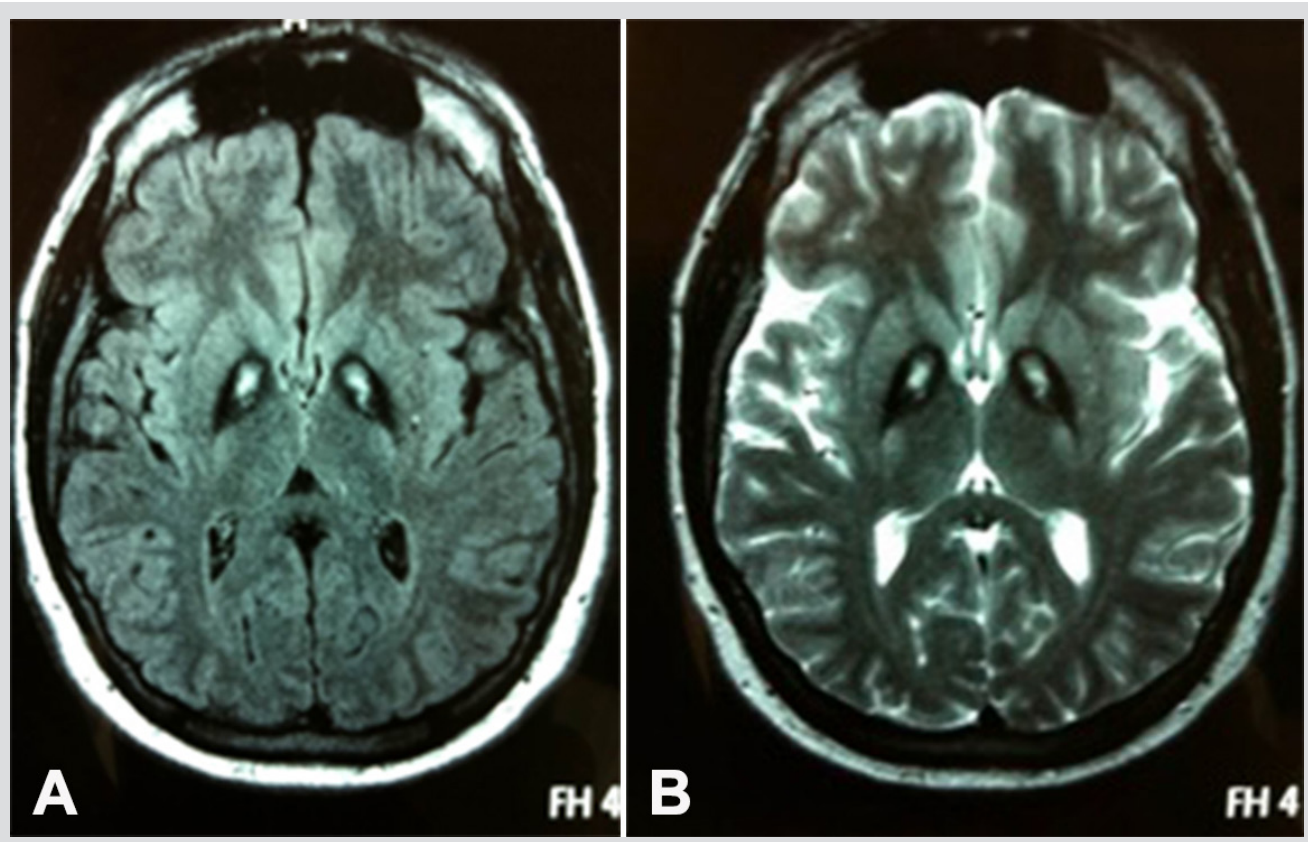

Figure 1.MRI: FLAIR (A) and T2 (B). Bilateral, symmetric foci of $\mathrm{T} 2$ hyperintensity were observed in the globus pallidus (GP), surrounded by pallidal T2 hypointensity

\section{DISCUSSION}

The patient presented with mild extrapyramidal signs and problems keeping up in school starting during childhood, and was considered to have a syndromic intellectual disability. Nevertheless, the progressive neurodegeneration over time made us re-evaluate this diagnosis. The presence of the so-called "Eye-of-the-tiger" sign represents a specific MRI pattern, a key diagnostic feature of NBIA, which appears as bilateral, symmetrical, central foci of hyperintense signals in the anteromedial GP, with a surrounding zone of hypointensity in the GP on T2W MR scanning. The central T2 relatively hyperintense spot or line within the globi pallidi is due to gliosis and vacuolization ${ }^{[2]}$. One of the proposed hypotheses of brain iron accumulation is that aberrant oxidation of lipofuscin to neuromelanin and insufficient cysteine dioxygenase lead to abnormal accumulation. Of note, iron deposition can be seen in normal aged brains and has been described in other neurological entities, including heredodegenerative and storage diseases.

However, the presence of this imaging sign does not guarantee that a PanK2 enzyme mutation will be found on analysis, as other mutations leading to this may be involved ${ }^{[3]}$. One study found that patients who have mutations that lower the residual activity of the PanK2 enzyme are more likely to develop PKAN between the ages of 1 and $6^{[4]}$, as in our case.

Clinically, the spectrum is wide and includes: extrapyramidal symptoms, dystonia, tremors, significant speech disturbances, dysphagia, dementia (present in most patients with PKAN), visual impairment (optic atrophy and retinal degeneration), seizures, akathisia[Q6] and neuropsychiatric dysfunction ${ }^{[2]}$.

PKAN has some differential diagnoses including Wilson's disease, hepatitis, drug toxicity and Huntington's disease ${ }^{[5]}$. 
According to the time of onset, PKAN has been classified as early-onset (classic) or late-onset (atypical). This case was considered to have an "atypical" presentation, taking into account the delay of extrapyramidal dysfunction for several years, the more gradual progression of the disease process, spasticity and psychiatric disturbances. Of note, prominent speech defects also dominate in the atypical form.

The treatment of patients with PKAN is mostly symptomatic. The Food and Drug Administration (FDA) approved a clinical trial examining the use of the iron chelator deferiprone for $\mathrm{NBIA}^{[6]}$, even though an iron chelator has been previously shown to be ineffective. Levodopa and anticholinergic medications may be helpful in treating parkinsonian symptoms; baclofen in moderate doses relieves the stiffness and spasms and can reduce dystonia; benzodiazepines have been used for choreoathetotic movements. Methscopolamine bromide can be attempted for excessive salivation. Deep brain stimulation (DBS) may be considered in severe cases of NBIA.

\section{CONCLUSION}

Atypical PKAN is a challenging diagnosis that requires a multidisciplinary team approach as it can be easily misdiagnosed.

\section{REFERENCES}

1. Salomão RPA, Pedroso JL, Gama MTD, Dutra LA, Maciel RH, Godeiro-Junior C, et al. A diagnostic approach for neurodegeneration with brain iron accumulation: clinical features, genetics and brain imaging. Arq Neuropsiquiatr 2016;74:587-596.

2. Bokhari MR, Zulfiqar H, Bokhari SRA. Hallervorden Spatz disease (pantothenate kinase-associated neurodegeneration, PKAN). [Updated 2019 Dec 12]. In: StatPearls [Internet], Treasure Island (FL): StatPearls Publishing; 2019. Available from: https://www.ncbi.nlm.nih.gov/books/NBK430689/.

3. Goldberg M, Malik K, Jiswant R, Khaku AS. Eye-of-the-tiger sign in neurodegeneration with brain iron accumulation. Cureus 2019;11(6):e4936.

4. Hartig M, Hortnagel K, Garavaglia B, Zorzi G, Kmiec T, Klopstock T, et al. Genotypic and phenotypic spectrum of PANK2 mutations in patients with neurodegeneration with brain iron accumulation. Ann Neurol 2006;59:248-256.

5. Hosseinialhashemi M, Daneshfard B, Hashemi A. "Eye of the Tiger" in a non-responsive neuropsychiatric patient: a case report. Acta Med Iran 2018;56(1):71-73.

6. ClinialTrials.gov [Internet]. Ferrochelating treatment in patients affected by neurodegeneration with brain iron accumulation (NBIA) [September 2018]. Available from: https:// clinicaltrials.gov/ct2/show/NCT00907283. 